\title{
XXXVI. Ueber neuere nordamerikanische Edelstein- Vorkommen.
}

\author{
Von \\ G. Kunz in Hoboken, N. J.
}

D i a mant. Das Vorkommen des Diamantes in Wisconsin wurde von Herrn H. Nichols in Minneapolis, Minn., entdeckt. Derselbe hat im Sommer 1887 mit einigen Herren im Plum Creek, Bezirk Rock Elm, Pearce County, Wisc., eine Untersuchung auf Gold angestellt. Bei dieser Gelegenheit wurde durch einen Arbeiter im Sande des Flussufers einige Fuss unter dem Wasserspiegel ein glänzender Stein gefunden, welcher als Diamant erkannt wurde. Im weiteren Verlaufe der Arbeiten wurden noch einige Krystalle entdeckt und auch später durch Herrn Ne well drei Meilen flussaufwärts ein ebensolcher ausgewaschen.

Als im Jahre 1888 mit Waschen von Gold begonnen wurde, wurden in ganz kurzer Zeit noch vier weilere Diamanten, einer an der Oberfläche der Kiesablagerung und einer in ungefähr sechs Fuss Tiefe aufgefunden. Auch im westlichen Arme des Plum Greek förderte $1889 \mathrm{Hr}$. Nichols einige Diamanten zu Tage, sowohl aus dem Kiese, wie aus den gewaschenen Antheilen des Goldsandes. Gold findet sich sowohl in allen Hauptarmen des Plum Creek, als auch längs seiner kleineren Zuflüsse.

Herr Nichols hat mir nun sowohl Proben des goldhaltigen Sandes, als auch drei Diamanten selbst zur Untersuchung eingesendet. Die letzteren wogen 160,5, 46 und 19,5 mg. Der grösste Krystall war ein Hexakisoktaëder mit gerundeten Flächen, farblos mit schwach grünlichem Ton und konnte zu einem vollkommenen Brillanten von $\frac{3}{16}$ Karat verschliffen werden. Der zweitgrösste bildet ein etwas in die Länge gezogenes Hexakisoktaëder von schwach gelblicher Farbe. Die Oberfläche ist nicht so glatt wie die des grössten und ist völlig bedeckt mit kleinen krystallinischen Eindrücken. Der dritte ist ein elliptischer Hexakisoktaëder-Zwilling von matter Oberfläche, weiss mit einem schwach gelblichen Tone. 
Die mikroskopische Untersuchung des goldführenden Sandes liess, ausser Quarzkörnchen, erkennen: Magnetit, Ilmenit, Almandin in Körnern und winzigen, scharfen Dodekaëdern ; sehr kleine, durchscheinende, glänzende Kryställchen, wabrscheinlich Hessonit oder Spessartin ; endlich abgerollte Krystalle und Körner von Monazit. Ein kleines Korn, welches als Platin angesprochen wurde, ging verloren, ehe es genauer gepruft werden konnte. Der Sand gleicht also den goldfübrenden Sanden von Burke County, N. C. und von Hall County, Ga.

So interessant die Auffindung dieser neuen Diamantfundstelle ist, so ist es doch fraglich, ob diese Goldsande ergiebiger und eine grössere commercielle Bedeutung erlangen werden, als die Goldsande von Nord-Carolina und Georgia bisher erlangt haben.

Sapphir findet man in Montana an den unter folgenden Namen bekannten Localitäten : El Dorado Bar, Spokane Bar, French Bar, Ruby Bar, ungefähr sechs Meilen längs des Missouri-Flusses gelegen, und in ihren beiden äussersten Punkten etwa sechszehn Meilen westlich von Helena entfernt. Der ungefähre Mittelpunkt bildet die zwölf Meilen westlich von Helena bei Stubb's Ferry gelegene Spokane Bar.

Systematische Versuche, diese Sandbänke auf Sapphire auszubeuten, sind bis jetzt nicht gemacht, wohl aber sind dieselben gründlich nach Gold durchsucht worden. Erst in neuerer Zeit ist man auf diese Sapphire, von welchen gelegentlich einzelne in die grösseren Städte gelangten, aufmerksam geworden, veranlasst durch den hohen Preis und die Nachfrage nach Steinen von so ausgesprochenen Farben wie Rubin und Sapphir. Im Jahre 4889 erwarb daher eine Gesellschaft eine sehr grosse Landstrecke, um den Abbau dieser Bänke in Angriff zu nehmen. Die Ausbeute an sich wäre keine geringe und zeigen die gefundenen Steine eine grosse Mannigfaltigkeit der Farben: roth, gelb, blau und grun, aber leider nur in lichteren Tönen. Besonders ausgesprochen ist die letztere Farbe, aber auch wieder mehr blaugrun als smaragdgrün. Nahezu alle Steine zeigen in geschliffenem Zustande einen fast metallähnlichen Glanz, welcher als ganz besonders charkteristisch für das Mineral dieser Localitäten angesehen werden kann. Wirklich tief gefärbte Rubine und Sappbire sind nicht gefunden worden.

Die Sapphire aller dieser Localitäten längs des Missouri finden sich in einer Schicht goldführenden Glacialkieses von einigen Zoll Dicke, welche direct auf einem schieferigen Gesteine aufliegt. In der Ablagerung an der Ruby Bar wurde auch ein Mastodonzahn in der Sapphirschicht gefunden.

Unter den Begleitmineralien wurden beobachtet: farbloser Topas in schönen, bis zu $\frac{1}{4}$ Zoll langen Krystallen, ähnlich denen vom Thomas Mountain in Utah; Granat in Körnern hin und wieder bis zu Erbsengrösse und von so schön rubinrother Farbe, dass sie fälschlich für Rubine gehalten worden sind; Gyanit in zerbrochenen, durchseheinenden Krystallen mit 
blauen Flecken; Zinnerz in abgerollten, concentrischen Massen bis $\frac{1}{4}$ Zoll Durchmeser; Limonit in Pseudomorphosen nach Pyrit und in concentrischen Massen von radialer Structur; Chalcedon in kleinen unregelmässigen Stücken und abgerollte Calcitbrocken.

Die Arbeiten an der Ruby Bar haben einen Gang von Eruptivgestein aufgedeckt, welcher das Schiefergestein, auf dem der glaciale Goldkies ruht, durchbricht. Dasselbe ist nach H. A. Miers ein blasiger GlimmerAugit-Andesit mit reichlich vorhandenem braunem Glimmer und porphyrischen Krystallen von Augit. Die Grundmasse besteht hauptsächlich aus Feldspathmikrolithen mit viel Zwischenklemmungsmasse und viel Magnetit und aus mit einem braunen Glase erfülten Hohlräumen. Letzteres wird mit grüngelber Farbe durchsichtig und zeigt spbärolithische Structur. In diesem Eruptivgesteine wurden Krystalle von Sapphir, Pyrop und Granat gefunden. Es kann daher nicht zweifelhaft sein, dass die ursprüngliche Quelle aller Sapphire in den Sandbänken längs des Missouri in einem nun zerstörten, dem oben beschriebenen ähnlichen Gesteine zu suchen ist. Augenscheinlich sind nördlich von diesen Bänken einige Kämme dieses Gesteines durch Gletscher erodirt worden, von welcher Localität aber ist nicht bekannt. Jedenfalls nicht von Ruby Bar, weil diese Lagerstätte sechs Meilen südlich von der El Dorado Bar gelegen, wo eine Quantitat Sapphire gefunden worden sind; eher noch von einigen nun nicht mehr vorhandenen oder uberdeckten Gängen nördlich der El Dorado Bar. Bergbau in dieser Gegend wird gewiss noch eine Anzabl Gănge zu Tage fördern, wie es bei Ruby Bar der Fall war beim Treiben eines Versuchsbaues einige hundert Fuss von dem Punkte, wo das Ausstreichen des ursprünglichen vier Fuss starken Ganges beobachtet worden war.

Türkis. Proben dieses Minerals von einem Vorkommen bei Poncha City, Saguache County, Col., wurden mir von Herrn Thi ele in Pueblo zugesendet. Es sind nierige Massen bis zu $\frac{1}{2}$ Zoll Durchmesser oder dünne Adern von blaugrüner, offenbar von Verwitterung herrührender Farbe. Das Vorkommen ist völlig übereinstimmend mit jenem von Los Cerrillos, N. M.

Zwei weitere neue Fundorte wurden in den Burro Mountains in der Nähe der alten Paschal Smelting Works, 15 Meilen südwestlich von Silver City, Grant County, N. M., durch Herrn G. W. Waring aus Silver City entdeckt.

Der Türkis durchsetzt das Gestein in Adern und Schnüren von wechselnder Dicke. Ein 50 Fuss hoher Trümmerhaufen und zahlreiche kleine, verwitterte Türkisfragmente zeigen, dass diese Localität wie die ubrigen neumexicanischen bereits von den Ureinwohnern in ausgedehnter Weise bearbeitet worden ist. Auch fand sich ungefähr zwölf Meilen von dieser Fundstelle eine indianische Begräbnissstätte, woselbst in jedem Grabe, das 
geöfinet wurde, einige polirte oder unregelmässig geformte Türkise angetroffen wurden.

Im Jahre 1890 wurden auch wieder Ausbeutungsversuche in der unter dem Namen "Castilian turquoise Mine" bekannten Grube angestellt. Dieselbe liegt sieben Meilen von Los Cerrillos, N. M. Eine zweite Grube liegt an dem Wege von diesem Orte nach Santa Fé etwa eine balbe Meile von Bonanza und grenzt an die erstgenannte an. In beiden Gruben ist, wie zabllose sich vorfindende Steinhämmer beweisen, von den Indianern viel gearbeitet worden. Diese Grube wurde während der letzten Jahre geöffnet und ein Schacht auf 75 Fuss Tiefe eingebracht und bei etwa 40 Fuss Tiefe die Fundstätte angetroffen. Dieselbe streicht nahezu von Ost nach West. Das Gestein ist das gleiche wie in der alten Grube, die Farbe des. Türkis aber eine bessere. Während der acht Monate dauernden Versuchsarbeiten wurden einige tausend Steine gefunden, zum Theil von hübsch blauer Farbe, und obschon nicht ganz an die persischen hinanreichend, immerhin von genúgend guter Qualität, um verarbeitet zu werden.

Je ein weiterer Fundort für Türkis wurde, wie mir Herr T. A. Hiestand von Manitou, Col., mittheilt, ungefähr $2 \frac{1}{2}$ Meile sudwestlich von Riverside, Arizona, sowie an einer Localität in den Pinal Mountains, zwei Meilen südlich von Bloody Tanks auf einem Bergrücken zwischen Globe und Silverton, Arizona, aufgefunden. Der Bergrucken ist auf eine Länge von mehr als einer halben Meile gestreift durch Türkisadern von grüner Farbe und zeigt ringsherûm zablreiche Spuren prähistorischer Thätigkeit.

Asterisirender Quarz wurde im letzten Jahre durch Steinhändler in Ottawa verkauft. Derselbe ist durchsichtig, farblos und zeigt, in bestimmter Richtung geschnitten, bei starker Beleuchtung einen auffallenden Asterismus.

Rauch quar z. Schöne Krystalle bis zu $3 \frac{1}{2}$ Zoll Länge wurden im Three Mile Gulch, drei Meilen stidöstlich von Helena, Mont., aufgefunden.

Hydrolith. Dünne Ghalcedonschalen, von $\frac{1}{2}$ bis $1 \frac{1}{2}$ Zoll Durchmesser, gefüllt mit Wasser und einer beweglichen Libelle, werden ziemlich häufig gefunden an der Küste von Oregon bei der Yuaquina-Bay und Astoria.

Holzachat. Von den bekannten drei verkieselten sogen. "Wäldern “ im Chalcedony-Park an der Atlantic- und Pacific-Eisenbahn ist der nächstgelegene ungefähr eine Quadratmeile gross und eingeschlossen von einem $50^{\prime}$ bis $100^{\prime}$ hohen Tafellande. Beinahe alles verkieselte Holz liegt auf der flachen Ebene unter diesem Tafellande und ruht auf Schichten von Sandstein. Die untere Schicht ist chocoladefarben, eine andere weiss, eine weitere schwarz und eine andere, ein compacter Sandstein, grau und auf diesem rubt eine Schicht von weissem Sandstein, in welchem ursprünglich alles fossile Holz dieser Localität gefunden wurde. Durch Abwaschung und Verwitterung dieser Formation rollten die Stämme nach der tiefer gelegenen 
Ebene. Keiner der Stämme aus dem oberen Lager ist in aufrechter Stellung, noch sind irgend welche Wurzeln sichtbar, und da keiner dieser Bäume irgend eine Spur der ursprünglichen Rinde zeigt, scheint es sehr wahrscheinlich, dass das gesammte Lager einst das Bett eines Inlandmeeres oder Sees gewesen war.

Zwei weitere Lager verkieselten Holzes befinden sich, das eine 8, das andere 16 Meilen vom Chalcedony-Park entfernt und sind an zahlreichen Stellen längs der Atlantic- und Pacific-Bahn entblösst. Die Qualität des Holzachats ist aber nicht so fein wie die in den drei ursprunglichen Lagern.

Ein kleiner fossiler Wald, dessen verkieseltes Holz sehr mit jenem des Chalcedony-Parkes ubereinstimmt, befindet sich etwa drei Meilen von Los Cerrillos, N. M.

Opal. Im Jahre 1890 fand Herr J. Allen aus Yonkers, N. Y., Feueropale in einem Haufen Gestein, welches aus einer Grube in einer Tiefe von 22 Fuss herausgeschlagen worden war, bei der Farm von William Leasure bei Whelan, 20 Meilen südwestlich von Colfax, fast an der Grenze der Staaten Whashington und Idaho. Das dortige Opalvorkommen ist ein mehr oder weniger reichliches, da die letzten vier Fuss des Gesteines mit Edelopal erfúllte Hohlräume enthielten. Diese Varietät findet sich in einem blasigen, stark zersetzten Basalt in Stucken von Erbsen- bis zu Hühnereigrösse. Die kleineren Stückchen sind farbenreich, während die grösseren wenig oder kein Farbenspiel zeigen. Die Qualität einzelner untersuchter Stucke erwies sich als sehr fein, so dass, wenn das Material so reichlich ist wie angegeben, es das vielversprechendste unserer Edelsteinvorkommen sein wird.

Hy a lit. Grosse Mengen durchsichtigen Hyalits wurden in Lake County, Gol., aufgefunden. Ferner im Trappgestein bei den Fullen des WillametteFlusses bei Oregon City, Ore., und in traubigen Massen ähnlich denen von Waltsch, in Böhmen im Weiser Valley Lavafeld, ungefähr 20 Meilen nördlich von Weiser, Idaho. 